\title{
Maxillofacial Radiology 167
}

SADJ February 2019, Vol. 74 No. 1 p43

\section{CJ Nortjé}

Below are two cases of a tumour rather commonly found in other bones of the body but rarely seen in the jaws. When it occurs in the maxillofacial region it is most frequently in the 50-60 year-old age group. What are the important radiological features and what is your diagnosis?

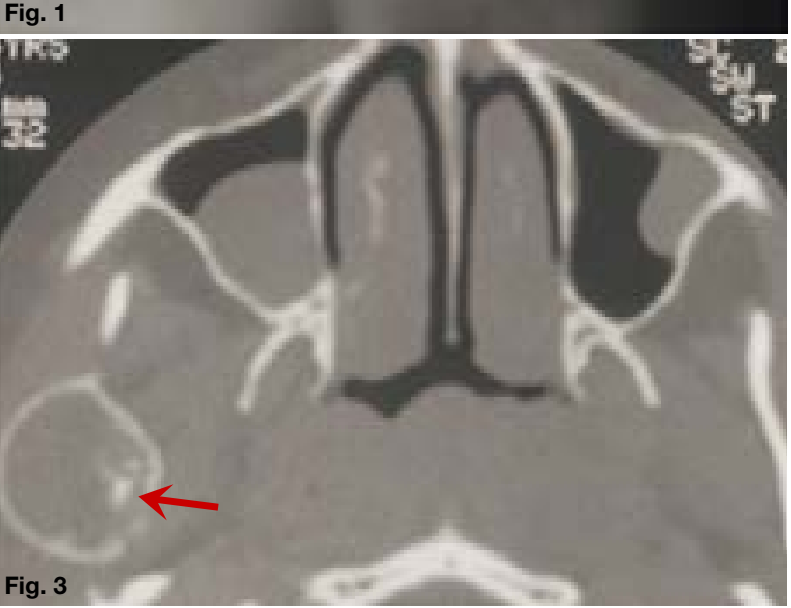

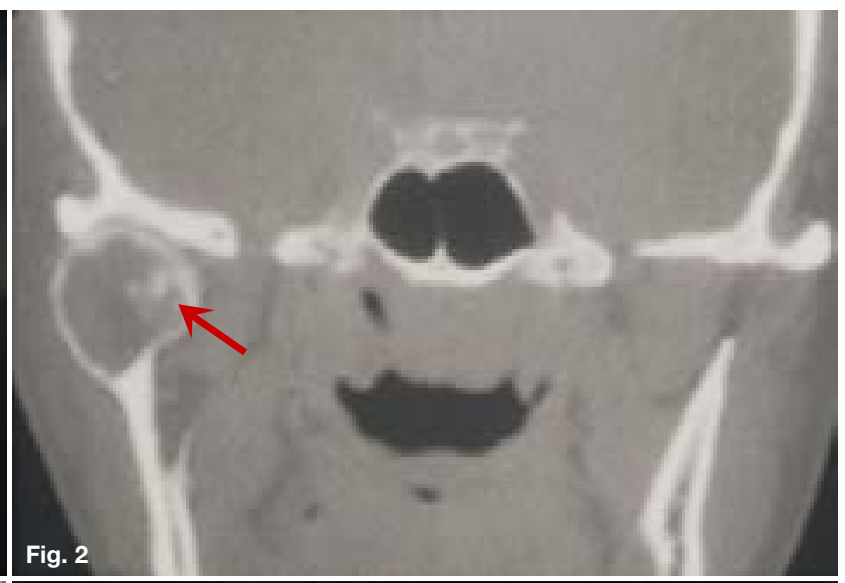

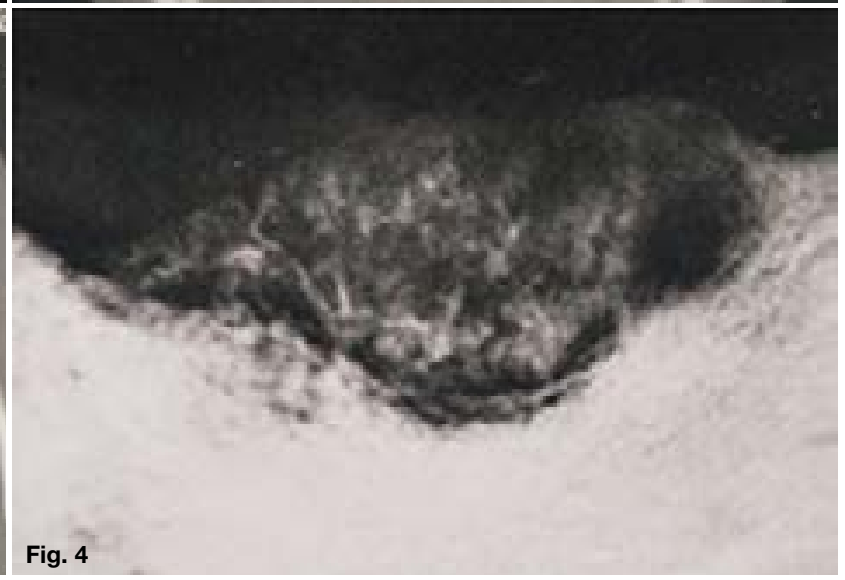

\section{INTERPRETATION}

Figure1 shows an expansive lesion with well-defined borders. Figures $2 \& 3$ are coronal and axial CT images of the same lesion showing irregular calcifications (arrows) within the radiolucency, histologically diagnosed as a chondroma, a benign tumour of cartilage without any bone formation. On occasion, the chondroma may become partially ossified and is then more appropriately called an osteochondroma (see MFR case 113), a slow growing, locally invasive, tumour of bone and cartilage. There is some laxity in the use of the terms as well as some difficulty in separating a partially ossified chondroma from the osteochondroma. Malignant forms of chondroma are termed chondrosarcoma. There are two types of chondroma. The central chondroma arises deep in the bone (Figures 1, $2 \& 3$ ), expanding the cortical plates. It is probably more common than the periosteal chondroma, which develops on the surface. Radiologically, the periosteal type may show an irregular, variably radiopaque, and radiolucent mottled lesion protruding from the cortex (Figure 4) as a raised radiolucent mass in which there are scattered and irregular areas of calcification.

CJ Nortjé: $B C h D, P h D, A B O M R, D S c$. Faculty of Dentistry, University of the Western Cape. E-mail: cnortje@uwc.ac.za
Beneath is a saucer-shaped concavity of the alveolar ridge, probably produced by pressure erosion. The base of the concavity is sclerotic, which suggests the benign nature of the lesion. Due to the paucity of published cases no definite features of the periosteal chondroma are presently available. Although the chondroma is a rather common type of bone tumour it is rarely found in the jaws. In the mandible the posterior part, especially the coronoid and condylar processes are areas of predilection. The anterior part of the maxilla is the site of nearly all the chondromas affecting the upper jaw, where they tend to extend into the nose and paranasal sinuses. When the condyle is affected, an early symptom may be a slight discomfort in the joint region followed by a progressive deviation of the jaw to the unaffected side and eventually restrictions in jaw movements. Resection is usually the preferred treatment because of the aggressive nature and tendency of the tumour to recur. Radiopacities present in the maxillary sinuses on the coronal and axial CT images are suggestive of mucus retention cysts.

\section{References}

Stafne EC \& Gibilisco JA: Oral Roentgenographic Diagnosis, $4^{\text {th }}$ Ed, W.B Saunders. Philadelphia 1975 pp 196-197. 